\title{
Seeing the stolen starlight with Herschel
}

\author{
Haley L. Gomez ${ }^{1}$, Edward L. Gomez ${ }^{1,2}$ and Peter Hargrave ${ }^{1}$ \\ ${ }^{1}$ School of Physics and Astronomy, Cardiff University, \\ The Parade, Cardiff, UK, CF24 3AA \\ email: haley.gomez@astro.cf.ac.uk \\ email: p.hargrave@astro.cf.ac.uk \\ ${ }^{2}$ Las Cumbres Observatory Global Telescope, Inc., 6740 Cortona Dr, Suite 102, Goleta, CA \\ 93117, USA \\ email: egomez@lcogt.net
}

\begin{abstract}
The Herschel Space Observatory is ESA's fourth Cornerstone mission and will be the largest, most sensitive telescope ever put into space. It will be the first space observatory to observe from the far-infrared to the submillimetre waveband, unveiling the cool, hidden universe for the first time. Herschel will observe stars and galaxies at the stage of formation and discover where all the cosmic dust polluting galaxies comes from. Given the huge public interest in large space missions such as Hubble and Spitzer, Herschel is an ideal opportunity to excite and inform the UK public during the International Year of Astronomy 2009. Here we present some of the education and outreach projects created by the Herschel Outreach Group (HOG).
\end{abstract}

Keywords. submillimetre, telescopes, Herschel, education, outreach

\section{Herschel}

The universe is a very dusty place consisting of tiny particles of solid material floating around in the space between the stars. Cosmic dust is important in a number of astronomical processes as it enhances star formation and provides the building blocks for life. Unfortunately dust is also responsible for blocking out light from distant stars and galaxies, hiding star formation and active galaxies from our optical telescopes. Luckily this 'dusty' cloud has a silver lining: dust grains absorb visible light from stars, and the stolen starlight is recycled at longer wavelengths, shining in the infrared and submillimetre parts of the electromagnetic spectrum (between 10-1000 $\mu \mathrm{m}$ ). Unlike optical telescopes which have been in use for hundreds of years, observing this stolen starlight has only become possible during the last few decades, with recent space missions such as IRAS, ISO and Spitzer, and the ground cameras SCUBA and BLAST (e.g. Fig. 1) throwing up a few surprises. One major surprise is how much infrared and submillimetre light we observe from sources beyond our own Milky Way. This light is known as the extragalactic background (Fig. 2a) and comparing the total light shining in the visible wavebands (stars, blue), right through to submillimetre wavelengths (dust, red) we see an unexpected result: the amount of energy shining in our universe from stars and dust is almost the same. This hints that dust grains are responsible for stealing (and re-radiating at longer wavelengths) almost half of the total visible light radiated from stars and galaxies since the Big Bang.

Current submillimetre detectors are ground-based or balloon experiments, yet this long wavelength light is very difficult to detect from the ground due to our atmosphere. The Herschel Space Observatory is designed to combat this problem and is particularly unique as it observes very cold $\left(-253^{\circ} \mathrm{C}\right)$ dust in the far-infrared and submillimetre (specifically at $55-672 \mu \mathrm{m})$. As ESA's fourth cornerstone mission, it will be the only space telescope to 


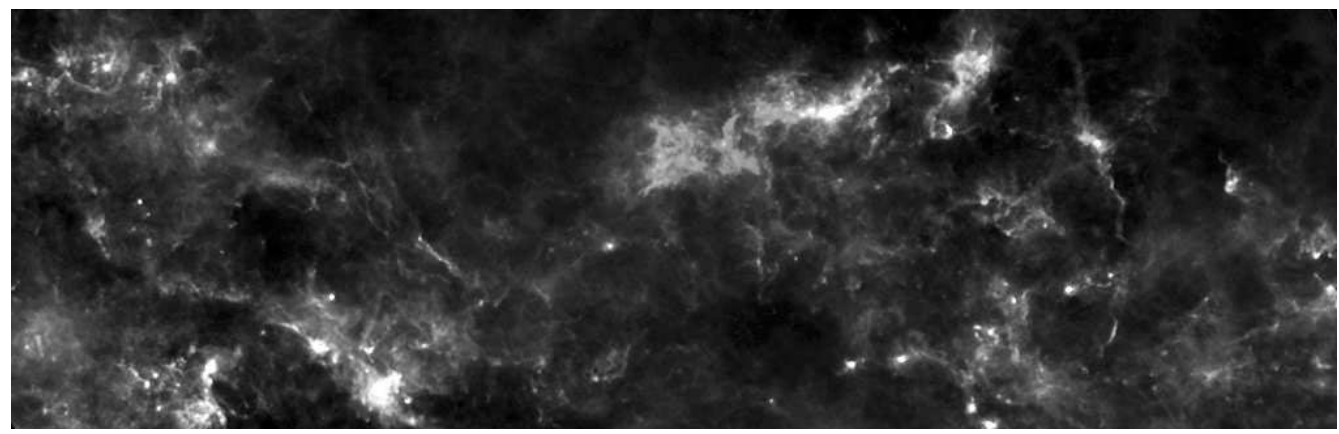

Figure 1. The balloon-borne experiment BLAST has started to probe the elusive submillimetre waveband. Here is the BLAST submillimetre image of the Vela nebula (credit BLAST consortium). This is the kind of striking image we expect to see with Herschel's SPIRE camera.
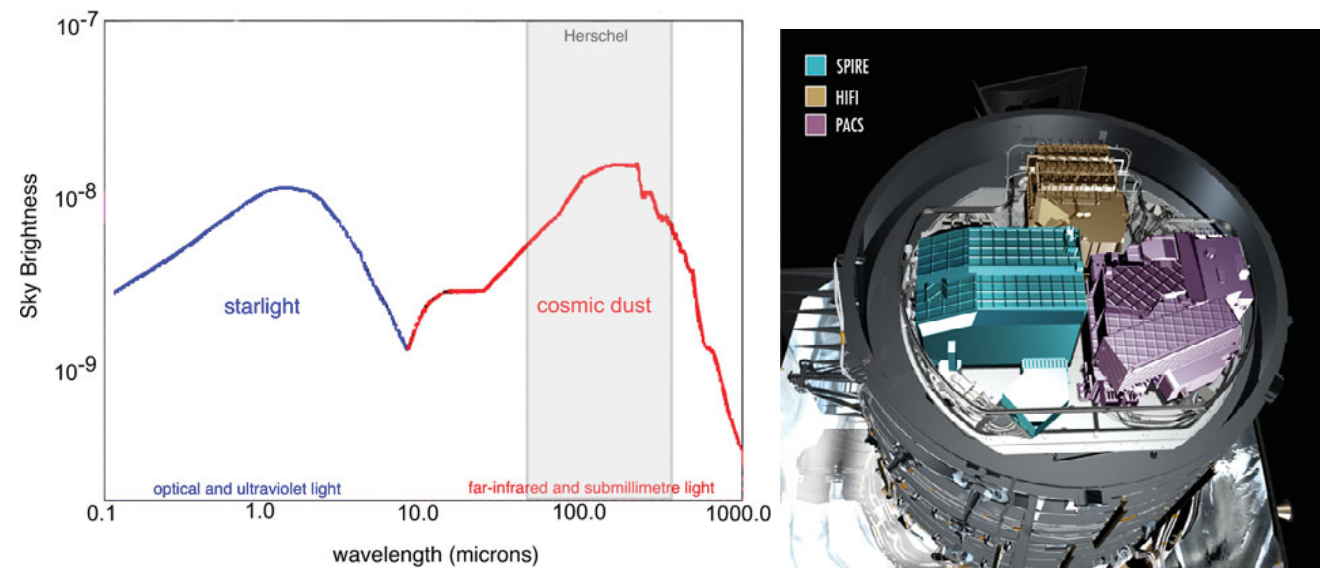

Figure 2. (a) The extragalactic background made up of radiation from distant objects beyond our own Milky Way (adapted from Dole et al. (2006)). The solid lines show the radiation from visible light (blue) and cosmic dust (red). The grey shaded region shows the wavelengths at which Herschel will observe. (b) Herschel's instruments: the HIFI (Heterodyne Instrument for the Far Infrared) spectrometer; and the PACS (Photoconductor Array Camera and Spectrometer) and SPIRE (Spectral and Photometric Imaging REceiver) cameras and imaging spectrometers (credit ESA).

see light beyond $200 \mu \mathrm{m}$ (shaded region Fig. 2a) and will have the largest mirror launched into space (1.5 times larger than Hubble's dish). Herschel has three powerful instruments on-board (Fig. 2b): SPIRE, PACS and HIFI and uses super-cooled technology, operating at temperatures down to only a few tenths of a degree above absolute zero $(-273 \mathrm{deg} \mathrm{C})$.

Some of the important questions Herschel will help answer are: how do stars and planetary systems form? How did galaxies form and evolve into the systems we see today? What can we learn about comets and other bodies in the Solar System? What can we learn about the molecular chemistry of the universe? We do not have to wait long for the answers: Herschel was launched from French Guiana on an Ariane-5 rocket in May 2009 along with the Planck satellite. Both missions designed to last for three years (when the cooling Helium runs out) with astronomers receiving data approximately six months after launch. During these three years, Herschel will literally see the universe in a different light. 

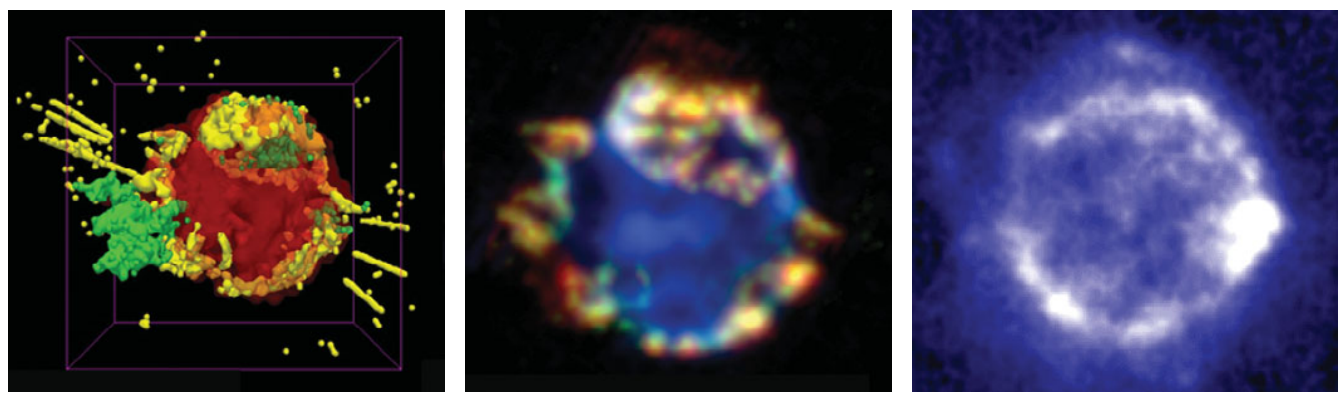

Figure 3. Infrared and submillimetre views of the historical supernova remnant Cas A. From left to right: 3-D reconstruction of hot silicon/argon (yellow) and hot iron (green) and cool debris (red) as seen by Spitzer and the Chandra Observatory (credit NASA/CXC/MIT/T.DeLaney). Multi-colour infra-red image of Cas A with Spitzer (Rho et al. 2008) showing the distribution of argon (green) and silicon gas (blue) and 10,000 Earth masses of warm dust (red). SCUBA's $850 \mu \mathrm{m}$ image of Cas A revealing a large mass of cold dust not seen by Spitzer (Dunne et al. 2003).

\section{Outreach and education activities}

Given the coincidence of the launch date, the heavy UK involvement and IYA09, Herschel provides many educational and public outreach (EPO) opportunities. To capitalise on this, we have formed, on a voluntary basis, the UK Herschel Outreach Group (HOG), chaired by Professor Matt Griffin (Principal Investigator of the SPIRE camera on Herschel), with membership comprised of Herschel scientists across the UK and EPO professionals from STFC, LCOGT, the Faulkes Telescope Project and Science Made Simple. The EPO activities designed by the HOG team include: an outreach website, social networking sites (facebook, twitter, wordpress), interactive shows to demonstrate Herschel science, public and school talks by Herschel scientists, art installations, exhibitions and education projects. Here we discuss the Smoking Supernova education project and our social networking activities. Further details on HOG EPO will be discussed elsewhere.

\subsection{The smoking supernova educational project}

Supernovae are the catastrophic explosions which occur at the evolutionary endpoints of massive stars. The explosion blasts material out into the surroundings at very high speeds, sweeping up the surrounding gas into a giant bubble. The shell or bubble is bright at many different wavelengths with different physical mechanisms responsible for each; e.g. in X-ray light, we are looking at extremely hot (millions of degrees) supernova gas compared to the gas shining in the visible (thousands of degrees).

Supernovae have recently become a hot topic in galactic astronomy as recent evidence suggests they may be responsible for creating lots of cosmic dust. We used the Spitzer telescope and SCUBA to observe the supernova remnant, Cassiopeia A (Cas A, Fig. 3) located at a distance of 11,000 light years from Earth. When we turned our infrared (Spitzer) and submillimetre (SCUBA) eyes onto Cas A to look for warm dust (Rho et al. 2008) and cold dust respectively (Dunne et al. 2003, 2009; Morgan et al. 2003), we found evidence that exploding stars like Cas A could be responsible for littering our universe with dust grains. However, there could be other mechanisms responsible and we need to observe supernovae from space to test this theory.

Herschel will unlock the mystery of the origin of dust in the universe, creating an opportunity for an exciting education resource. The Smoking Supernova project will allow users to piece together the multi-wavelength puzzle, where each piece is a different wave- 
length: X-ray (very hot gas), visible (hot gas), infra-red (warm dust) and submillimetre (cold dust). The activity pack will include the datasets and instructions to: (i) make a 3-colour image of Cas A using different wavebands (an interactive online version is already available at faulkes-telescope.com); (ii) investigate how the structure in the different colours/wavebands differ; (iii) measure the radius of the blast wave and use this to estimate how long ago the star exploded and (iv) find out how much dust was formed in the supernovae. Users will then help astronomers balance the dust-budget sheets in galaxies.

\subsection{Social networking: become a fan}

Over the past 5 years, social networking has becoming increasingly popular as a way of keeping in touch with other users and sharing multimedia web content with friends via the internet. Its success is based on the novel 'sharing' aspect of networking; it provides people with a way to share interests with a self-selected group and users can connect with others based on relationships or common ideals. Consequently, social networking sites are used by millions making this a powerful medium for EPO activities. According to the internet surveying organization comScore (Jan 2009), there are 890 million webusers worldwide, 550 million of which are members of a social networking site; $62 \%$ of these are clustered around a relatively low number of sites, mainly facebook, Bebo and MySpace. By taking advantage of these pre-existing media, astronomers can massively promote their EPO activities. Indeed, the addictive nature of the social network experience makes it the the perfect place to promote missions like Herschel; these networks readily spread information to large audiences and uniquely, allow users to 'belong' to a scientific community (Gomez, Gomez \& Yardley 2009). To capitalise on the popularity of facebook, we have created a Herschel Astronomy Page (with approximately 300 fans to-date) to keep interested parties up-to-date with launch dates, events and blogs (written by Dave Clements). Anyone can now become a fan of Herschel on facebook.

Herschel is the first space observatory with the capabilities to resolve questions on the origin, nature and quantity of cold dust in the universe. The coincidence of this ESA launch with IYA2009 will not only ensure significant media coverage but combined with our multi-format HOG projects, will also maximise our chances of inspiring the next generation of scientists and engineers. Astronomers and the public alike are eagerly awaiting results from Herschel; one thing is certain, there will be many more dusty surprises in store.

\section{References}

Dole, H., Lagache, G., Puget, J.-L., Caputi, K. I., Fernández-Conde, N., Le Floc'h, E., Papovich, C., Pérez-González, P. G., Rieke, G. H. \& Blaylock, M. 2006, A\& A, 451, 417

Dunne, L., Eales, S., Ivison, R., Morgan, H. \& Edmunds, M. 2003, Nature, 424, 285

Dunne, L., Maddox, S.J., Ivison, R.J., Rudnick, L.R., DeLaney, T.M., Matthews, B.C., Crowe, C.M., Gomez, H.L., Eales, S.A. \& Dye, S. 2003, MNRAS, 394, 1307

Gomez, E., Gomez H., \& Yardley, J. 2009, DotAstronomy and the New Media, R.J. Simpson \& D. Ward-Thomspon (eds) (Bristol: Canopus Academic Publishing) p.i75

Morgan H. Dunne L., Eales S., Ivison R. \& Edmunds M.G. 2003, ApJ, 597, L33

Rho, J., Kozasa, T., Reach, W.T., Smith, J.D., Rudnick, L., DeLaney, T., Ennis, J.A., Gomez, H. \& Tappe, A. 2008, ApJ, 673, 271 